\section{0-gauge and 23-gauge phacovitrectomy for idiopathic macular holes: comparison of complications and long-term outcomes}

R Krishnan, C Tossounis and Y Fung Yang
Department of Ophthalmology, Queen Alexandra Hospital, Portsmouth, UK

Correspondence: R Krishnan, Department of Ophthalmology, Queen Alexandra Hospital, Portsmouth, Hants PO6 3LY, UK

Tel/Fax: +44 (0)2392 286444 E-mail: radhikrishnan2004@ yahoo.co.uk

Received: 12 July 2012 Accepted in revised form: 26 September 2012 Published online: 16 November 2012

Presented as a poster at the 2012 Annual Congress of Royal College of Ophthalmologists in Liverpool.

\begin{abstract}
Purpose To assess and compare the complications and long-term visual and anatomical outcomes of 20- and 23-gauge phacovitrectomy (PVT) for idiopathic macular hole repair.

Methods A retrospective, consecutive, interventional case series of 72 eyes with idiopathic macular holes were managed with 20-gauge PVT $(n=36)$ or 23-gauge PVT $(n=36)$. All patients underwent a posteriorchamber intra-ocular lens implantation and internal-limiting-membrane peel with the aid of either trypan blue or brilliant

blue $\mathrm{G}_{250}$ dyes.

Results The macular hole closure rate was 97\% with 20 -gauge PVT and $100 \%$ with 23-gauge PVT. Mean follow-up was 24.06 ( \pm 12.83) months with 20-gauge PVT and 17.47 ( \pm 5.79 ) with 23-gauge PVT. Mean visual acuity improvement in LogMar was 0.41 ( \pm 0.32$)$ with 20-gauge PVT and 0.52 $( \pm 0.25)$ with 23 -gauge PVT $(P=0.135)$. The incidence of retinal breaks was higher with the 20-gauge PVT compared with the 23-gauge PVT, occurring in $13(36.1 \%)$ and $4 \mathbf{( 1 1 . 1 \% )}$ cases, respectively $(P=0.025)$. There were no cases of retinal detachment. Postoperative intraocular pressure (IOP), at day 1, was significantly higher with 20-gauge PVT at $24.77( \pm 12.68) \mathrm{mm} \mathrm{Hg}$ compared with $17.20( \pm 6.95) \mathrm{mm} \mathrm{Hg}$ in 23-gauge PVT $(P=0.0028)$. Operating times were longer in 20-gauge PVT at $89.36( \pm 15.26) \mathrm{min}$ compared with the 23-gauge PVT at 79.33 $( \pm 23.82)$ min $(P=0.04)$.

Conclusion The 20-gauge PVT compared with 23-gauge PVT for macular hole repair is
\end{abstract}

a slightly longer procedure and was associated with an increased incidence of retinal breaks and short-term elevated IOP. Both techniques have comparable visual and anatomical outcomes, but the 23-gauge PVT has advantages with regard to safety profile and operating time.

Eye (2013) 27, 72-77; doi:10.1038/eye.2012.227; published online 16 November 2012

Keywords: vitrectomy; macular hole; vitreous; retina; surgery

Introduction

Vitrectomy with intravitreal gas tamponade is now the established treatment for idiopathic macular holes since it was first described by Kelly and Wendel in 1991. ${ }^{1-3}$ Cataract progression is a frequent consequence following this procedure with all cases requiring cataract surgery within 2 years of vitrectomy. ${ }^{4}$ Combined cataract and macular hole surgery eliminates the need for a second surgical procedure allowing earlier visual rehabilitation and has shown good long-term outcomes while still providing good anatomical and visual results. ${ }^{5-7}$ Furthermore, removal of the crystalline lens and replacing it with a thinner intraocular lens implant can allow a more complete vitrectomy and a greater intraocular gas fill.

With advancement in vitreoretinal technology, a variety of vitreoretinal systems offering 25-, 23-, and 20-gauge transconjunctival entry is available with their differing advantages. The 23-gauge vitrectomy was created to provide the advantages of smaller 
sutureless incision surgery combined with sturdier instruments to allow easier manipulation of the globe compared with 25 -gauge instrumentation. ${ }^{8}$ However, 25-gauge instruments continue to evolve and have improved significantly since their introduction. ${ }^{9}$ From the surgical standpoint, 23-gauge instrumentation may benefit from a more favourable learning curve giving a similar 'feel' to 20-gauge systems. In addition, it also allows sufficient intraocular illumination for internallimiting membrane (ILM) peeling without the need for a separated brighter light source as required with 25-gauge systems.

The 23-gauge vitrectomy appears to have a good safety profile compared with 20-gauge systems and good visual outcomes for a number of different posterior segment conditions. ${ }^{10,11}$ However, there have been no studies comparing the efficacy of different vitrectomy gauge systems when used by a single surgeon in treating a specific pathology.

The aim of this study is to evaluate the safety profile and short- and long-term visual and anatomical outcomes of 20-gauge phacovitrectomy (PVT) compared with 23-gauge PVT for idiopathic macular holes in a single surgeon series.

\section{Materials and methods}

Consecutive patients who underwent macular hole surgery (pars plana vitrectomy, ILM peel and gas) combined with phacoemulsification and insertion of a posterior chamber intraocular lens implant (PCIOL) by a single surgeon were identified and retrospectively reviewed. All surgery was performed in a single unit over a 5-year period.

Surgery was performed under peribulbar local anaesthesia or general anaesthesia. Phacoemulsification was performed through a sutureless clear corneal incision followed by an IOL into the capsular bag. Where there was a breach of the posterior capsule, the IOL was inserted into the sulcus. In the 20-gauge PVT, localised conjunctival peritomies were performed and a $20 \mathrm{~g}$ microvitreoretinal (MVR) blade was used to create the three pars plana sclerotomies, $3.5 \mathrm{~mm}$ from the limbus, into the vitreous cavity. The 20-gauge instruments were used including the Eckhardt ILM peeling vitreoretinal forceps (DORC International, Zuidland, Netherlands). For 23-gauge PVT, the conjunctiva was displaced towards the limbus and held in position with a pressure plate. A 23-gauge MVR blade was used to create a shelved scleral incision and the $23-\mathrm{g}$ valved microcannulae (DORC International) was inserted using a trocar. The microcannula was left in place providing a channel for the 23-gauge instruments to be inserted into and out of the eye.
In both groups, a core and peripheral vitrectomy was performed following induction of a posterior vitreous detachment if not already present. Visible vitreous base was trimmed with the vitrector but not specifically indented for shaving. An ILM peel was performed in all cases with the assistance of trypan blue (MembraneBlue; DORC International) or brilliant blue $\mathrm{G}_{250}$ (ILM Blue; DORC International). If present, then the epiretinal membrane (ERM) was peeled before ILM. The membrane-peeling forceps was held in the surgeon's right hand in all cases.

A search for iatrogenic breaks was performed towards the end of the procedure with scleral indentation. Any breaks or suspicious areas were treated with cryotherapy or argon laser retinopexy. Fluid-gas exchange was performed and a non-expansile mixture of perfluoropropane $\left(\mathrm{C}_{3} \mathrm{~F}_{8}\right)$ was used as tamponade in all cases.

The postoperative treatment regime was guttae chloramphenicol $0.5 \%$ four times a day and dexamethasone $0.1 \%$ four times a day. No routine anti-ocular hypertensive medication was given. Patients were advised to posture face-down for 10 days.

\section{Outcome measures and statistics}

Outcomes that were recorded included patient demographics, grade of macular hole, preoperative best-corrected visual acuity (BCVA) and postoperative BCVA at 6 months, 12 months, and final follow-up, postoperative closure of the macular hole at 6 months and 12 months, complications during surgery, length of surgery and postoperative intraocular pressure (IOP).

BCVA was recorded as a Snellen visual acuity and converted to LogMar for analysis. The groups were compared using Student's t-test and Fisher's exact test as appropriate. The threshold for significance was $P<0.05$ in all calculations.

\section{Results}

Seventy-two consecutive eyes of seventy-two patients (thirty-six eyes underwent 20-gauge PVT and thirty-six eyes underwent 23-gauge PVT) were identified for the study. The demographic and preoperative clinical features of the two groups were similar (Table 1).

Seventy-one of the seventy-two eyes in this study had a minimum follow-up period of 11 months extending up to 63 months. One patient in the 20-gauge PVT group was noted to have an open macular hole 2 months after surgery following complete reabsorption of the gas tamponade. Further surgery was offered but the patient declined and was therefore discharged at 5.5 months. The mean follow-up was higher in the 20-gauge PVT 
Table 1 Demographic and preoperative clinical features of the 20G and 23G phacovitrectomy (PVT) groups

\begin{tabular}{|c|c|c|c|c|}
\hline Type of PVT & $20 G$ & $23 G$ & Total & P-value \\
\hline Mean age (years) & 69 & 68.64 & & 0.80 \\
\hline \multicolumn{5}{|l|}{ Gender } \\
\hline Male & $11(30.6 \%)$ & $7(20 \%)$ & $18(25.3 \%)$ & \\
\hline Female & $25(69.4 \%)$ & $28(80 \%)$ & $53(74.7 \%)$ & \\
\hline \multicolumn{5}{|l|}{ Site of surgery } \\
\hline OD & $17(47.2 \%)$ & $17(47.2 \%)$ & $34(47.2 \%)$ & \\
\hline OS & $19(52.8 \%)$ & $19(52.8 \%)$ & $38(52.8 \%)$ & \\
\hline $\begin{array}{l}\text { Mean duration of } \\
\text { MH (months) }\end{array}$ & 6.75 & 4.06 & & 0.0083 \\
\hline \multicolumn{5}{|l|}{ Grade of $M H$} \\
\hline II & $5(13.9 \%)$ & $6(16.7 \%)$ & $11(15.3 \%)$ & \\
\hline III & $23(63.9 \%)$ & $22(61.1 \%)$ & $45(62.5 \%)$ & \\
\hline IV & $5(13.9 \%)$ & $1(2.8 \%)$ & $6(8.3 \%)$ & \\
\hline NR & $3(8.3 \%)$ & $7(19.4 \%)$ & $10(13.9 \%)$ & \\
\hline \multicolumn{5}{|l|}{ Distortion } \\
\hline Yes & $30(83.3 \%)$ & $34(94.4 \%)$ & $64(88.9 \%)$ & \\
\hline No & $6(16.7 \%)$ & $1(2.8 \%)$ & $7(9.7 \%)$ & \\
\hline NR & 0 & $1(2.8 \%)$ & $1(1.4 \%)$ & \\
\hline \multicolumn{5}{|l|}{ Cataract } \\
\hline Mild & $16(44.4 \%)$ & $19(52.8 \%)$ & $35(48.6 \%)$ & \\
\hline Mod & $6(16.7 \%)$ & $4(11.1 \%)$ & $10(13.9 \%)$ & \\
\hline NR & $14(38.9 \%)$ & $13(36.1 \%)$ & $27(37.5 \%)$ & \\
\hline $\begin{array}{l}\text { Mean pre-op BCVA } \\
\text { (LogMar) }\end{array}$ & 0.86 & 0.89 & & 0.6383 \\
\hline
\end{tabular}

group at 24 months compared with 17.5 months in the 23-gauge PVT group $(P=0.0064)$.

Thirty-five of the thirty-six eyes in the 20-gauge PVT group had successful closure of the macular hole. All patients in the 23-gauge PVT group had successful closure of the macular hole. None of the patients in this study had reopening of a closed macular hole throughout the follow-up period.

Thirteen out of thirty-six (36\%) patients in the 20-gauge PVT group had one or more iatrogenic retinal breaks, all of which were at the entry site. Ten $(77 \%)$ of these iatrogenic breaks were at the entry site corresponding with the sclerotomy used for the insertion of membranepeeling forceps. In the 23-gauge PVT, there were 4 retinal breaks out of 36 (11.1\%). Two were entry site breaks, one of which corresponded with the sclerotomy used for the insertion of the membrane-peeling forceps. One was an inferior peripheral break due to PVD induction and one was a retinal break due to impact of the cutter on the retina. There was a significant difference between the higher incidence of retinal breaks in the 20-gauge PVT compared with the 23-gauge PVT group $(P=0.0246$, Fisher's exact test). There were no cases of retinal detachment at anytime during the surgery or the postoperative follow-up period.

Operating times were found to be shorter with 23-gauge PVT with a mean of 89.36 min compared with $79.33 \mathrm{~min}(P=0.04)$.

Postoperative IOP was significantly higher after 20-gauge PVT compared with 23-gauge PVT at day 1 $(P=0.0028)$ and just below significance at day 7 $(P=0.052)$. There were no cases of hypotony in either group. At 2-3 months, the IOP was very similar between the two groups $(P=0.954)$. In the 20 -gauge PVT group, one patient had elevated IOP postoperatively which did not respond to medical management and went on to have a trabeculectomy.

The posterior lens capsule was ruptured during phacoemulsification in three eyes $(4.2 \%)$, one in the 20-gauge PVT group and two in the 23-gauge PVT group. Posterior chamber intraocular lens implants were successfully placed in the ciliary sulcus in these eyes before proceeding with vitrectomy. One of these sulcus placed lenses in the 23-gauge PVT dislocated into the vitreous cavity 1 month after surgery. A further vitrectomy procedure was carried out in this eye to remove this lens and an anterior chamber lens implant was inserted. There was one case of zonular dialysis (in the 23-gauge PVT group) requiring insertion of a Morcher ring but there was no vitreous loss in this case. Intraoperative complications are summarised in Table 2.

In the 23-gauge PVT group, two patients had cystoid macular oedema (CMO) postoperatively. One patient declined further treatment for this. The other patient developed an ERM and underwent a repeat vitrectomy and ERM peel 1 year after the initial procedure.

\section{Discussion}

This is the first report comparing longer term outcomes of 20-gauge PVT and 23-gauge PVT for idiopathic macular hole in a single surgeon series. Our study shows no significant difference between the visual outcomes and anatomical outcomes between the two groups with closure rates of 97 and $100 \%$ in the 20-gauge PVT group and 23-gauge PVT group, respectively, including in the longer term. This was despite the duration of macular holes before surgery being significantly higher in the 20-gauge PVT group. The anatomical closure rates compare very favourably with those of other published series looking at PVT and intravitreal gas tamponade with closure rates of $89-100 \%, 12-14$ and vitrectomy alone with intravitreal gas tamponade demonstrating a closure rate of $96 \% .{ }^{14,15}$

The incidence of inducing iatrogenic retinal breaks in 20-gauge vitrectomy for macular hole (without ILM peeling) has been reported as $3.2 \%$ with a hole closure 
Table 2 Intraoperative and postoperative complications

\begin{tabular}{|c|c|c|c|c|}
\hline Intraoperative and postoperative complications & $20 G$ & $23 G$ & Total & P-value \\
\hline PC rupture & $1(2.8 \%)$ & $2(5.6 \%)$ & $3(4.2 \%)$ & \\
\hline \multicolumn{5}{|l|}{ Iatrogenic retinal tears } \\
\hline All sites & $13(36.1 \%)$ & $4(11.1 \%)$ & $17(23.6 \%)$ & 0.0246 \\
\hline Entry site $^{\mathrm{a}}$ & $10(27.8 \%)$ & $2(5.6 \%)$ & $12(16.7 \%)$ & 0.0065 \\
\hline Zonular dialysis & 0 & $1(2.8 \%)$ & $1(1.4 \%)$ & \\
\hline \multicolumn{5}{|c|}{ Postoperative mean IOP and standard deviation $(\mathrm{mm} \mathrm{Hg})$} \\
\hline Day 1 & $24.77( \pm 12.68)$ & $17.20( \pm 6.95)$ & & 0.0028 \\
\hline Day 7 & $20.97( \pm 8.61)$ & $17.58( \pm 5.59)$ & & 0.052 \\
\hline $2-3$ months & $16.78( \pm 4.40)$ & $16.83( \pm 3.73)$ & & 0.954 \\
\hline CMO & 0 & $2(5.6 \%)$ & $2(2.8 \%)$ & \\
\hline Total number of eyes & 36 & 36 & 72 & \\
\hline
\end{tabular}

${ }^{\mathrm{a}}$ Corresponding to membrane-peeling forceps.

rate of $80.6 \% .^{3}$ However, the retinal detachment rate in this study was $5.6 \%$, suggesting that some tears may not have been detected intraoperatively leading to the postoperative retinal detachment. The incidence of iatrogenic retinal breaks in 20-gauge vitrectomy (with ILM peeling) for macular holes is higher at $12.5-17 \%$ but can demonstrate a hole closure of $91-100 \% .{ }^{16,17}$ The occurrence of iatrogenic retinal breaks in 23-gauge vitrectomy alone for macular hole was demonstrated as $4.3-8 \%$ with a $92-96 \%$ hole closure rate. ${ }^{15,18}$

Macular hole surgery is associated with a higher incidence of causing iatrogenic retinal breaks than vitrectomy surgery for other vitreoretinal pathology, 19,20 presumed to be due to the surgical induction of a posterior vitreous detachment. Induction of the posterior vitreous detachment can cause a tractional retinal break usually near the vitreous base inferiorly. $^{21}$

ILM peeling is currently thought to be an effective adjunct to achieve a higher macular hole closure rate and to prevent late reopening. ${ }^{16,17}$ This involves inserting the relatively large membrane-peeling forceps through the sclerotomy and traversing the vitreous base. Insertion of the forceps can cause a localised retinal dialysis while withdrawal of the forceps can lead to a tractional retinal break at the entry site by pulling on the vitreous frill around the internal lip of the sclerotomy.

There is a significant difference in the incidence of iatrogenic retinal tears occurring at the entry site corresponding with the sclerotomy used for inserting the membrane-peeling forceps between 20-gauge and 23-gauge PVT groups $(P=0.0065)$. This difference may be due to the 23-gauge microcannulae, which remain within the sclerotomies for the duration of the surgery for the instruments to pass through. These may be long enough to traverse the vitreous base such that the vitreous gel is less likely to engage with the membrane-peeling forceps on its way into and out of the vitreous cavity.

IOP was higher in the 20-gauge PVT group at 1 day and 1 week but this was only significant between the two groups at day 1. IOP was managed medically in all but one case (in the 20-gauge PVT group), which went on to have a trabeculectomy.

Increased postoperative inflammation has been identified as a possible reason for performing sequential rather than combined surgery for macular hole. In our series, there was one case of recurrent uveitis postoperatively in the 23-gauge vitrectomy group but this was managed with topical steroids and the patient was on no treatment at the final follow-up. A study comparing vitrectomy and combined surgery for a variety of vitreoretinal conditions identified an increased risk of postoperative fibrinous uveitis in the combined surgery group. However, this was only statistically significant for proliferative diabetic retinopathy cases. ${ }^{22}$ In our study, there were no cases of postoperative fibrinous uveitis observed in either group.

Intraoperative complications during cataract surgery were comparable or lower in our series for posterior capsular rupture (4.2\%) and zonular dialysis (1.4\%) compared with large studies of vitrectomised eyes undergoing cataract surgery with posterior capsular rupture occurring in 5-9\% and zonular dialysis in $5 \% .^{23,24}$ In our series, CMO occurred postoperatively in two patients $(2.8 \%)$. In one of these cases, it was associated with an ERM and responded to peeling. The CMO rate in vitrectomy alone (without ILM peeling) was $1 \%$ in a large multicentre study. ${ }^{25} \mathrm{CMO}$ rates in vitrectomised eyes can be as high as $13.6 \%{ }^{26}$ However, this is greatly affected by the pre-existing pathology 
leading to the initial vitrectomy and diabetic status of the patient.

In the 23-gauge PVT group, $36 \%$ of patients required suturing of at least one port and it has been suggested that the time to perform vitrectomy is greater with a 23-gauge vitreous cutter compared with 20-gauge due to the narrower diameter. Despite this a comparison of operating times showed a difference of $\sim 10 \mathrm{~min}$ on average between the two groups $(P=0.04)$ with 23-gauge PVT being slightly quicker. While the 23-gauge system may prove more time efficient on scleral entry and wound closure, due to its smaller gauge, removal of the vitreous volume takes longer than in the 20-gauge system.

23-gauge PVT for macular holes appears to be a safer alternative to 20-gauge PVT in terms of inducing less entry site breaks. Visual and anatomical results show equal efficacy between the two groups with 23-gauge PVT requiring slightly less surgical time.

The limitations of this study include the small size and retrospective study design. However, by limiting the sample to only include surgical procedures performed for a single indication by a single surgeon in one unit this removes variations in operative techniques and technical skill between surgeons as a potential cause for the different outcomes between the groups. Furthermore, this study included complete follow-up data with no patients being lost to follow-up.

\section{Summary}

\section{What was known before}

- 20-gauge vitrectomy is the historical gold standard. 23-gauge vitrectomy is sutureless with minimal tissue injury and shorter surgery times.

\section{What this study adds}

- Both 20-gauge and 23-gauge phacovitrectomies (PVTs) have comparable visual and anatomical outcomes. 20-gauge PVT is associated with an increased incidence of retinal breaks, especially at entry sites, and short-term elevated intraocular pressure. 20-gauge PVT is a slightly longer procedure compared with 23-gauge PVT for combined macular hole and cataract surgery.

\section{Conflict of interest}

The authors declare no conflict of interest.

\section{References}

1 Kelly NE, Wendel RT. Vitreous surgery for idiopathic macular holes. Results of a pilot study. Arch Ophthalmol 1991; 109(5): 654-659.

2 Wendel RT, Patel AC, Kelly NE, Salzano TC, Wells JW, Novack GD. Vitreous surgery for macular holes. Ophthalmology 1993; 100(11): 1671-1676.
3 Ezra E, Gregor ZJ, Moorfields Macular Hole Study Group. Surgery for idiopathic full-thickness macular hole: two-year results of a randomized clinical trial comparing natural history, vitrectomy, and vitrectomy plus autologous serum: Moorfields Macular Hole Study Group Report no. 1. Arch Ophthalmol 2004; 122(2): 224-236.

4 Cheng L, Azen SP, El-Bradey MH, Scholz BM, Chaidhawangul S, Toyoguchi M et al. Duration of vitrectomy and postoperative cataract in the vitrectomy for macular hole study. Am J Ophthalmol 2001; 132(6): 881-887.

5 Lahey JM, Francis RR, Fong DS, Kearney JJ, Tanaka S. Combining phacoemulsification with vitrectomy for treatment of macular holes. Br J Ophthalmol 2002; 86: 876-878.

6 Rogers S, Madhusudhana KC, Kang HK, Luff AJ, Canning CR, Newsom RS. Combined phacovitrectomy for macular hole: long-term results. Ophthalmic Surg Lasers Imaging 2007; 38: 452-456.

7 Scott IU, Moraczewski AL, Smiddy WE, Flynn HW Jr, Feuer WJ. Long-term anatomic and visual acuity outcomes after initial anatomic success with macular hole surgery. Am J Ophthalmol 2003; 135: 633-640.

8 Eckardt C. Transconjunctival sutureless 23-gauge vitrectomy. Retina 2005; 25: 208-211.

9 Spirn MJ. Comparison of 25, 23 and 20-gauge vitrectomy. Curr Opin Ophthalmol 2009; 20(3): 195-199.

10 Fine HF, Iranmanesh R, Iturralde D, Spaide RF. Outcomes of 77 consecutive cases of 23-gauge transconjunctival vitrectomy surgery for posterior segment disease. Ophthalmology 2007; 114: 1197-1200.

11 Tewari A, Shah GK, Fang A. Visual outcomes with 23-gauge transconjunctival sutureless vitrectomy. Retina 2008; 28: 258-262.

12 Theocharis IP, Alexandridou A, Gili NJ, Tomic Z. Combined phacoemulsification and pars plana vitrectomy for macular hole treatment. Acta Ophthalmol Scand 2005; 83: $172-175$.

13 Gottlieb CC, Martin JA. Phacovitrectomy with internal limiting membrane peeling for idiopathic macular hole. Can J Ophthalmol 2002; 37(5): 277-282.

14 Muselier A, Dugas B, Burelle X, Passemard M, Hubert I, Mathieu B et al. Macular hole surgery and cataract extraction: combined vs consecutive surgery. Am J Ophthalmol 2010; 150: 387-391.

15 Kusuhara S, Ooto S, Kimura D, Itoi K, Mukuno H, Miyamoto $\mathrm{N}$ et al. Outcomes of 23- and 25-gauge transconjunctival sutureless vitrectomies for idiopathic macular holes. Br J Ophthalmol 2008; 92: 1261-1264.

16 Passemard M, Yakoubi Y, Muselier A, Hubert I, Guillaubey A, Bron AM et al. Long-term outcome of idiopathic macular hole surgery. Am J Ophthalmol 2010; 149: $120-126$.

17 Park DW, Sipperley JO, Sneed SR, Dugel PU, Jacobsen J. Macular hole surgery with internal-limiting membrane peeling and intravitreous air. Ophthalmology 1999; 106: 1392-1397.

18 Sanisoglu H, Sevim MS, Aktas B, Sevim S, Nohutcu A. Outcomes of 23-gauge pars plana vitrectomy and internal limiting membrane peeling with brilliant blue in macular hole. Clin Ophthalmol 2011; 5: 1177-1183.

19 Mochizuki Y, Kubota T, Hata Y, Miyazaki M, Suyama Y, Enaida $\mathrm{H}$ et al. Surgical results of combined pars plana vitrectomy, phacoemulsification, and intraocular lens implantation. Eur J Ophthalmol 2006; 16: 279-286. 
20 Okuda T, Nishimura A, Kobayashi A, Sugiyama K. Postoperative retinal break after 25-gauge transconjunctival sutureless vitrectomy. Graefes Arch Clin Exp Ophthalmol 2007; 245: 155-157.

21 Tabandeh H, Chaudhry NA, Smiddy WE. Retinal detachment associated with macular hole surgery: characteristics, mechanism, and outcomes. Retina 1999; 19: 281-286.

22 Treumer F, Bunse A, Rudolf M, Roider J. Pars plana vitrectomy, phacoemulsification and intraocular lens implantation. Comparison of clinical complications in a combined versus two-step surgical approach. Graefes Arch Clin Exp Ophthalmol 2006; 244: 808-815.

23 Banker AS, Freeman WR, Kim JW, Munguia D, Azen SP. Vision-threatening complications of surgery for full-thickness macular holes. Vitrectomy for Macular Hole Study Group. Ophthalmology 1997; 104(9): 1442-1452.

24 Pardo-Muñoz A, Muriel-Herrero A, Abraira V, Muriel A, Muñoz-Negrete FJ, Murube J. Phacoemulsification in previously vitrectomized patients: an analysis of the surgical results in 100 eyes as well as the factors contributing to the cataract formation. Eur J Ophthalmol 2006; 16(1): 52-59.

25 Szijarto Z, Haszonits B, Biró Z, Kovacs B. Phacoemulsification on previously vitrectomized eyes: results of a 10-year-period. Eur J Ophthalmol 2007; 17(4): 601-604.

26 Grusha YO, Masket S, Miller KM. Phacoemulsification and lens implantation after pars plana vitrectomy. Ophthalmology 1998; 105(2): 287-294. 